\title{
Kolmogorov entropy changes and cortical lateralization during complex problem solving task measured with EEG
}

\author{
Lian-Yi Zhang
}

School of Electrical Engineering, Shanghai Dianji University, Shanghai, China.

Email: D310zlyi@sohu.com

Received 25 July 2009; revised 2 September 2009; accepted 3 September 2009.

\begin{abstract}
The objective is to study changes in EEG time-domain Kolmogorov entropy and cortical lateralization of brain function areas during complex problem solving mental task in healthy human subjects. EEG data for healthy subjects are acquired during complex problem solving mental task using a net of 6 electrodes. The subject was given a nontrivial multiplication problem to solve and the signals were recorded for 10s during the task. Kolmogorov entropy values during the task were calculated. It was found that Kolmogorov entropy values were obviously greater in P4 channel (right) than ones in P3 channel (left) during complex problem solving task. It indicated that all subjects presented significant left parietal lateralization for the total frequency spectrum. These results suggest that it may be possible to noninvasively lateralize, and even eventually localize, cerebral regions essential for particular mental tasks from scalp EEG data.
\end{abstract}

Keywords: Wada Test; Cortical Lateralization; EEG; Brain Function Area; Complex Problem Solving

\section{INTRODUCTION}

Functional lateralization is an important organizing principle of the human brain. The left and right cortices have different specializations and each contributes to the performance of most cognitive tasks. The cortical lateralization can be confirmed through experiments such as injecting sodium amytal into a hemisphere (Wada test), measuring RT (Radioisotope Tracer), the split brain, hemispherectomy, and so on. Being invasive procedures, these traditional techniques are associated with some risk and discomfort. Because of this, in recent years, a burgeoning interest has developed in replacing traditional techniques with noninvasive measures. Some of these newer techniques, like the Wadatest, are based on "deactivation" of the cortex, such as repetitive transcranial magnetic stimulation [1], whereas other methods are based on structural imaging analyses [2]. However, the most promising novel noninvasive methods include direct measures of physiological activation. Some newer methods include event-related brain potentials, and whole-head magnetoencephalography. Neuroimaging studies (PET, FMRI, CT, SPECT) have also help localize lateralization effects in specific cortical areas [3,4]. All of these newer methods have variable limitations, and none have yet supplanted the Wadatest as the "old standard" for lateralizing cerebral dominance [5]. Among the aforementioned approaches, the most simple and least costly are based on scalp EEG measurements. Multichannel EEG devices are readily available in most clinical sites and could thus be used to replace the invasive traditional methods. A common practice in estimating human brain activity during performance of a mental task is also to process the electroencephalogram (EEG) in order to detect signal changes that could be related to mental processes.

The aim was to establish an EEG-based lateralization test. Here we presented initial results for four subjects examined in a complex problem solving task with 6channel EEG time-domain Kolmogorov entropy computations. It was found that Kolmogorov entropy values were obviously greater in P4 channel (right) than ones in P3 channel (left) during complex problem solving task. It indicated that the subjects presented significant left parietal lateralization for the total frequency spectrum. These results were similar to those from the Wadatest. The notation KE is used to emphasize that it is the Kolmogorov entropy values of the time-domain EEG data.

The rest of the paper is organized as follows. Section 2 explains the methods proposed in this paper. Experiment task and data collection are described in Section 3. Rresults is in Section 4. Conclusions and Discussions are given in Section 5.

\section{METHOD}

\subsection{Algorithm}

Kolmogorov entropy (KE) describes the rate at which information about the state of the dynamic process is lost with time. Known as metric entropy, divide phase space 
into D-dimensional hypercubes of content $e^{D}$. Let $P_{i_{0}, \cdots, i_{n}}$ be the probability that a trajectory is in hypercube $\mathrm{i} 0$ at $\mathrm{t}=0, \mathrm{i} 1$ at $\mathrm{t}=\mathrm{T}, \mathrm{i} 2$ at $\mathrm{t}=2 \mathrm{~T}$, etc. Then define

$$
K_{n}=-\sum_{i_{0}, \cdots i_{n}} P_{i_{0}, \cdots i_{n}} \ln P_{i_{0}, \cdots i_{n}}
$$

where $\mathrm{KN}+1-\mathrm{KN}$ is the information needed to predict which hypercube the trajectory will be in at $(\mathrm{n}+1) \mathrm{T}$ given trajectories up to $\mathrm{nT}$. The Kolmogorov entropy is then defined by

$$
K E \equiv \lim _{T \rightarrow 0} \lim _{e \rightarrow 0^{+}} \lim _{N \rightarrow \infty} \frac{1}{N T} \sum_{n=0}^{N-1}\left(K_{n+1}-K_{n}\right)
$$

The calculation of KE from a time series typically starts from reconstructing the system's trajectory in an embedding space. The EEG signals can reflect the state of brain activity. The EEG can be represented by projections of all variables in a multi-dimensional state space. Let $x_{i}, i=1, \cdots, N$ be a sample series of EEG. It is a discrete time series. Then, a m-dimensional time delay vector (in an $\mathrm{N}$-dimensional space) $\mathrm{X}(\mathrm{n})$ can be constructed as follows:

$$
X(n)=\{x(n), x(n+\tau), x(n+2 \tau), \cdots, x(n+m-1) \tau\}
$$

where $\tau$ is the time delay and $m$ is the embedding dimension of the system. Then we can calculate the correlation sum $\mathrm{Cm}(\mathrm{e})$ introduced by Grassberger and Procaccia [6]:

$$
\begin{aligned}
C_{m}\left(e, N_{m}\right)= & \frac{2}{N_{m}\left(N_{m}-1\right)} \sum_{i=1}^{N} \sum_{j=i+1}^{N} \Theta\left(e-\left\|x_{i}-x_{j}\right\|\right) \\
& N_{m}=N-(m-1) \tau
\end{aligned}
$$

where $\Theta$ is the Heaviside step function, $\Theta(x)=0$ if $x \leq 0$ and $\Theta(x)=1$ for $\mathrm{x}>0$. $\mathrm{e}$ is a given distance in a particular norm. If an attractor is present in the time series, the values $C_{m}\left(e, N_{m}\right)$ would satisfy $C_{m}\left(e, N_{m}\right) \propto e^{D}$, where $\mathrm{D}$ is the correlation dimension of the attractor and given by:

$$
\begin{gathered}
d_{m}\left(N_{m}, e\right)=\frac{\partial \ln C_{m}\left(e, N_{m}\right)}{\partial \ln e} \\
D=\lim _{e \rightarrow 0} \lim _{N_{m} \rightarrow \infty} d_{m}\left(N_{m}, e\right)
\end{gathered}
$$

If $e$ is small enough and $d_{m}$ does not vary with $m$, Kolmogorov entropy (KE) can be calculated by the following equation:

$$
K E=\lim _{e \rightarrow 0} \lim _{m \rightarrow \infty} \frac{1}{\tau} \log \left(\frac{C_{m}(e)}{C_{m+1}(e)}\right)
$$

Here $\tau$ is the delay time. Higher and finite positive KE suggests chaos.
Actually, EEG signal is always changing with time. So the KE of EEG is not a constant over time. To measure the unorderly degree of EEG signal, mean Kolmogorov entropy within one second was introduced:

$$
\text { Mean } \quad K E=\frac{1}{N} \sum_{n=1}^{N} K E(n)
$$

In the following of this paper the time series indicated EEG time series and KE refered the mean KE of EEG.

\subsection{Complex Problem Solveing Task}

EEG data were acquired during the task using a net of 6 electrodes. The electrodes were placed at $\mathrm{O} 1, \mathrm{O} 2, \mathrm{P} 3, \mathrm{P} 4$, $\mathrm{C} 3$ and $\mathrm{C} 4$ reference to the 10-20 system to record the EEG data in the experiment. Recordings were made with reference to the $\mathrm{A} 1$ and $\mathrm{A} 2$, electrode by using a high-pass filter of $0.1 \mathrm{~Hz}$ and a low-pass fillter of $100 \mathrm{~Hz}$. Figure 1 shows the placement of electrodes. The impedances of all electrodes were kept below $5 \mathrm{~K} \Omega$. The EEG was acquired with a sampling rate of $250 \mathrm{~Hz}$, that is, 250 samples/second. The signals were recorded for 10 s during the task, so each segment gave 2500 samples per channel. The data were recorded using an IBM-AT controlling a Lab Master analog to digital converter with 12 bits of accuracy.

Data for four subjects were used for this study. The subjects are mail. Subjects 1 and 2 were employees of a university. Subject 1 was left-handed and aged 48. Subject 2 was right-handed and aged 39. Subject 3 and subject 4 were right-handed college students. Subjects were placed in a dim, sound controlled room. The subject was given a nontrivial multiplication problem to solve and, as in all of the tasks, was instructed not to vocalize or make over movements while solving the problem.

\section{RESULTS AND DISCUSSIONS}

In order to obtain the data of spontaneous EEG signals, a FIR with bandpass filter $0.5-30 \mathrm{~Hz}$ was used. At the beginning of calculation KE of four subjects' EEG signal piece by piece, first 4 seconds (1000 samples) data are chosen as basic data and step length is 25 samples (the samples within $0.1 \mathrm{~s}$ ). To compare the effects of left hemisphere and right hemisphere during different mental

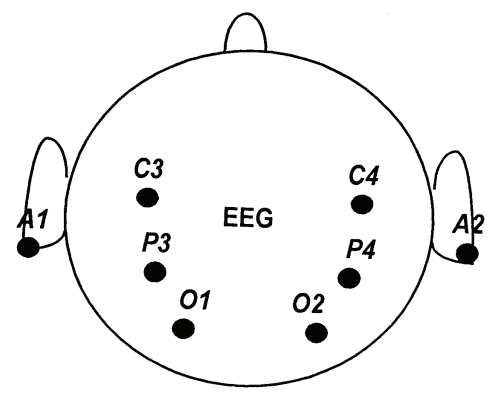

Figure 1. Electrode placement. 
tasks, the mean KE of $5^{\text {th }}, 6^{\text {th }}, 7^{\text {th }}, 8^{\text {th }}, 9^{\text {th }}, 10^{\text {th }}$ second of every subject in different channel are calculated respectively. For the same time interval and the same subject, the left channel's mean KE and the right channel's mean $\mathrm{KE}$ of each cortical function area consist of a pair of data. So for each brain function area (Central, Parietal and Occipital) under the mental task, every subject has 6 pair of KE data.

The properties of KE for different types of dynamics are: $\mathrm{KE}=0$ implies an ordered system, $\mathrm{KE}=\infty$ corresponds to a totally stochastic situation. The higher the $\mathrm{KE}$, the closer to a stochastic the system is. So for the bilateral of the same cortical function, the small value of KE corresponds to the dominant hemisphere.

Figure 2 shows the 24 pair of KE data in parietal area for all subjects during the task. From Figure 2, it can be seen that the KEs in P4 channel (right) are obviously greater than that in P3 channel (left). It means that all subjects presented significant left parietal lateralization for the total frequency spectrum $\left(\bar{d}=2.0714, \mathrm{~S}_{\mathrm{d}}=3.352, \mathrm{n}=24, \mathrm{t}=3.0274,0.01<\mathrm{p}<0.05\right)$. It can also be found from Figure 2 that sometime the mean $\mathrm{KE}$ on the right (P4) is smaller than that on the left (P3) and this indicate that sometime the right half may be the dominant hemisphere.

There is no significant diference of KE changes in the central area and occipital area. It may mean that there is no significant lateralization in the central area and occipital area.

There is no significant diference of KE changes for right-handed and left-handed in the experiment. It may mean that there is no significant lateralization in central, parietal and occipital brain function area during the mental task for right-handed and left-handed.

Despite their low spatial resolution, electrophysiological measurements have succeeded in showing accurately the time-course of stimulus processing in the human brain. Inparticular, the early phases of cortical processing can be detected by EEG or MEG techniques alone [6]. The changes in EEG time-domain Kolmogorov entropy (KE) and localization of related cortical areas during the complex problem solving mental task in human subjects.

The mean KE on the left (P4) is not always greater than that on the right (P3). Sometime the mean KE of the dominant hemisphere is larger than that of the nonominant. It means that sometime the right half may be the dominant hemisphere. That is to say, the dominant hemisphere is not always the same one during the same task. This indicates that the dominant hemisphere is not always the one that actuall controls performance on a particular task. This is consistent with previous known studies [7].

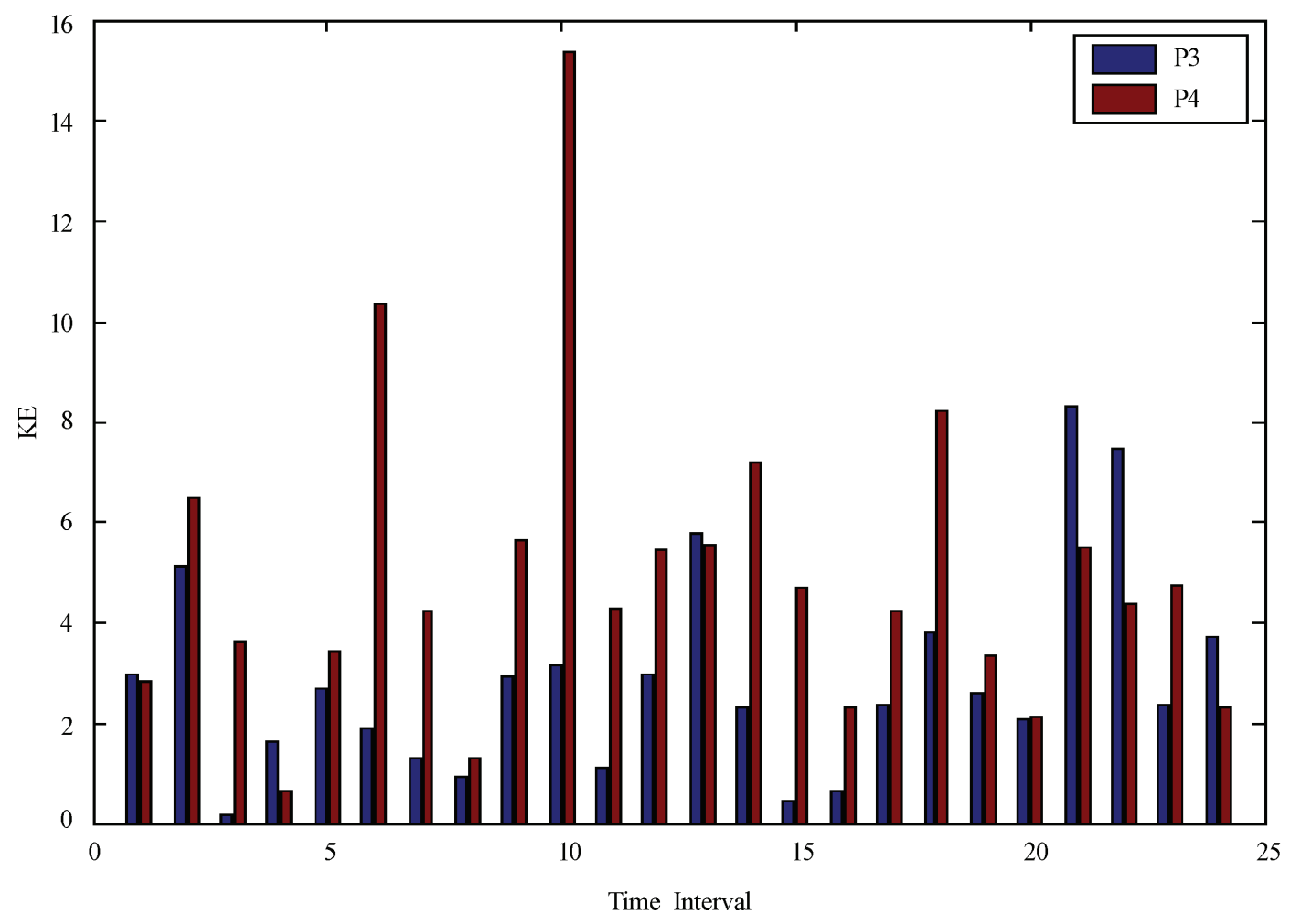

Figure 2. Comparison of KE between $\mathrm{P} 3$ and $\mathrm{P} 4$ during the task. 
When the value mean $\mathrm{KE}$ on the left (P3) is contrasted with the one on the right (P4), the difference is very apparent and the advantage is not always the same. This may means that the advantage of dominant hemisphere is always changing. The greater the difference, the more advantageous the dominant hemisphere.

There may be no significant lateralization diference of $\mathrm{KE}$ changes for right-handed and left-handed in central, parietal and occipital brain function area during the task. It may indicate that the difference between right-handed and left-handed is not always existentent in different brain function areas and in different mental tasks.

\section{CONCLUSIONS}

During the complex problem solving mental task, the subjects presented significant left parietal lateralization for the total frequency spectrum. There is no significant lateralization in the central and occipital area during the task. The dominant hemisphere is not always the same one during the same task. The lateralization determined by Kolmogorov entropy of EEG proposed in this paper is consistent with previous known studies. The Klmogorov entropy changes of EEG can describe the cortical lateralization.

The lateralization for some particular mental task may involve in several brain areas synchronously. It is invasive, convenient and useful to analyze and to localize the different brain function area with Kolmogorov entropy of EEG.

Our results suggested lateralization of the complex problem solving task area to the left hemisphere. The results reported here do not replace the results obtained with the Wada test and other techniquies, but supplement them. In summary, these results suggest that it may be possible to noninvasively lateralize, and even eventually localize, cerebral regions essential for particular mental tasks from scalp EEG data. This could be very helpful in presurgical planning. These findings are preliminary and need to be further studied in a large population base.

\section{REFERENCES}

[1] A. Pascual-Leone, J. R. Gates, A. Dhuna. (1991) Induction speech arrest and counting errors with rapid-rate transcranial magnetic stimulation $[\mathrm{J}]$. Neurology, 41, 697-702.

[2] P. D. Charles, Abou-KhalilR, Abou-KhalilB, et al. (1994) MRI asymmetries and language dominance [J]. Neurology, 44, 2050-2054.

[3] J. E. Adock, R. G. Wise, J. M. Oxbury et al. (2003) Quantitative fMRI assessment of the differences in lateralization of language-related brain activation in patients with temporal lobe epilepsy [J]. Neuroimage, 18(2), 423-438.

[4] P. Brockway and P. John. (2005) fMRI may replace the Wada test for language lateralization/localization [J]. Neuroimage, 11(5), S277.

[5] C. Ramon, M. Holmes, J. F. Walter, et al. (2009) Power spectral density changes and language lateralization during covert object naming tasks measured with high-density EEG recordings [J]. Epilepsy\&Behavior, 14, 54-59.

[6] P. Grassberger and I. Procaccia. (1983) Measuring the strangeness of strange attractors, Physica, 9D, 189-209,.

[7] C. Spironelli and A. Angrilli. (2009) Developmental aspects of automatic word processing: Language lateralization of early ERP components in children, young adults and middle-aged subjects [J]. Biological Psychology, 80, $35-45$.

[8] J. B. Hellige. (1993) Hemispheric asymmetry: What's right and what's left $[\mathrm{M}]$. Cambridge, MA: Harvard University Press. 